\title{
Experimental chronic entrapment of the sciatic nerve in adult hamsters: an ultrastructural and morphometric study
}

\footnotetext{
R.A.D. Prinz ${ }^{1}$,

M. Nakamura-Pereira ${ }^{1}$,

B. De-Ary-Pires ${ }^{1}$,

D.S. Fernandes ${ }^{1}$,

B.D.S.V. Fabião-Gomes ${ }^{1}$,

P.S. Bunn ${ }^{1}$,

A.M.B. Martinez ${ }^{2}$,

M.A. Pires-Neto ${ }^{1}$

and R. Ary-Pires ${ }^{1}$
}

Departamentos de ${ }^{1}$ Anatomia and ${ }^{2}$ Histologia e Embriologia, Universidade Federal do Rio de Janeiro, Rio de Janeiro, RJ, Brasil

\section{Correspondence \\ R. Ary-Pires \\ Departamento de Anatomia \\ UFRJ, CCS, Bloco F \\ 21941-590 Rio de Janeiro, RJ \\ Brasil \\ Fax: +55-21-2553-6321 \\ E-mail: arypires@anato.ufrj.br}

Research supported by FAPER], CNPq, CAPES and TWAS.

Received October 2, 2002

Accepted May 9, 2003

\begin{abstract}
Entrapment neuropathy is a group of clinical disorders involving compression of a peripheral nerve and interference with nerve function mostly through traction injury. We have investigated the chronic compression of peripheral nerves as an experimental procedure for detecting changes in ultrastructural nerve morphology. Adult hamsters (Mesocricetus auratus, $\mathrm{N}=30$ ) were anesthetized with a $25 \%$ pentobarbital solution and received a cuff around the right sciatic nerve. Left sciatic nerves were not operated (control group). Animals survived for varying times (up to 15 weeks), after which they were sacrificed and both sciatic nerves were immediately fixed with a paraformaldehyde solution. Experimental nerves were divided into segments based upon their distance from the site of compression (proximal, entrapment and distal). Semithin and ultrathin sections were obtained and examined by light and electron microscopy. Ultrastructural changes were qualitatively described and data from semithin sections were morphometrically analyzed both in control and in compressed nerves. We observed endoneurial edema along with both perineurial and endoneurial thickening and also the existence of whorled cell-sparse structures (Renaut bodies) in the subperineurial space of compressed sciatic nerves. Morphometric analyses of myelinated axons at the compression sites displayed a remarkable increase in the number of small axons (up to 60\%) in comparison with the control axonal number. The distal segment of compressed nerves presented a distinct decrease in axon number (up to 40\%) comparatively to the control group. The present experimental model of nerve entrapment in adult hamsters was shown to promote consistent histopathologic alterations analogous to those found in chronic compressive neuropathies.
\end{abstract}

Key words

- Nerve entrapment

- Ultrastructure

- Morphometry

- Hamster

- Renaut body 
Entrapment neuropathies are produced by existing anatomical arrangements that cause compression or constriction of a peripheral nerve. The three most common entrapment syndromes (the thoracic outlet syndrome, the carpal tunnel syndrome, and the cubital entrapment syndrome) are remarkable examples of the dramatic clinical features with motor and sensory symptoms often associated with autonomic dysfunction. They occur at specific places where nerves are confined to narrow anatomic pathways and therefore are particularly susceptible to constricting pressures. In those neuropathies the neural lesion is directly attributable to local causes comprising disturbances to nerve vascular supply (1), axonal transport mechanisms $(2,3)$, degeneration of myelin sheaths (4), axonal damage (5), and/or connective tissue changes such as endoneurial edema (6). Nerves can be damaged in a number of ways such as: a) ischemia; b) physical agents such as traction, pressure stretching, cold or heat; c) infectious and inflammatory processes; d) ingestion of drugs and metals; e) infiltration by or pressure from tumors; $f$ ) the effects of systemic disease; g) compression and/or section. In the present study we investigated by light and electron microscopy the ultrastructural changes of peripheral nerve lesions induced by chronic compression using an experimental model for detecting tissue changes promoted by entrapment.

All experimental procedures involving animal use were done in full agreement with the Animal Care and Use Committee of the Instituto de Biofísica, Universidade Federal do Rio de Janeiro. Adult hamsters (Mesocricetus auratus, $\mathrm{N}=30$ ) were deeply anesthetized with a $25 \%$ pentobarbital solution and each animal received a surgically applied small silicone cuff embracing the right sciatic nerve. The left sciatic nerves were not operated and represented the control group. After surgery, animals were kept under veterinary care for variable survival intervals (2-15 weeks), after which they were sacri- ficed by ether inhalation, being immediately perfused transcardially with $4 \%$ paraformaldehyde. The nerves were then dissected and divided into segments based upon their distance from the site of nerve compression (proximal, entrapment and distal). These segments were then immersed in $2 \%$ glutaraldehyde in $0.1 \mathrm{M}$ cacodylate buffer, $\mathrm{pH} 7.4$, for $2 \mathrm{~h}$ followed by $1 \%$ osmium tetroxide with $0.8 \%$ potassium ferricyanide and $\mathrm{CaCl}_{2}$ in $0.1 \mathrm{M}$ cacodylate buffer, $\mathrm{pH}$ 7.4, for $1 \mathrm{~h}$. Nerve blocks were subsequently dehydrated in graded acetone solutions and embedded in PolyBed 812. Semithin (500 nm) and ultrathin $(60-70 \mathrm{~nm})$ sections were stained with toluidine blue, uranyl acetate and lead citrate, respectively. Ultrathin sections were examined under a Zeiss EM-900 (Carl Zeiss, Oberkochen, Germany) electron microscope. Semithin sections of both control and compressed nerves were photographed with a light microscope (Zeiss-Axioscope) and then analyzed morphometrically (Image Pro Plus software package, Media Cybernetics, Carlsbad, CA, USA) and central nerve regions were compared to marginal nerve regions in each group. The central region of the sciatic nerve was defined as the inner circular zone corresponding to $50 \%$ of the nerve diameter, surrounded by the marginal region corresponding to $25 \%$ of the nerve diameter. Myelinated axons in each nerve region were systematically counted with the use of a uniform sampled area $\left(3600 \mu \mathrm{m}^{2}\right.$ grid) and the data are respectively expressed as percentage of axon number (mean $\pm \mathrm{SEM}$ ). It was assumed that axon number in the central or marginal regions of the control nerves represented $100 \%$ of the normal axon population in each respective region. Statistical analysis was performed with a computerized package (SPSS 11.0 for Windows) and consisted of bivariate followed by univariate analysis of variance. The Duncan multiple range test was used to compare the experimental groups $(\mathrm{P}<0.01)$.

To our knowledge the present results 
represent the first observations of experimental nerve entrapment in adult hamsters. After surgery, the main pathologic findings were already thoroughly established after a 5-week survival interval and most alterations followed a similar pattern of presentation in animals enduring longer compression (up to 15 weeks). Ultrastructural nerve morphology exhibited endoneurial edema, with thickening of both the endoneurium and perineurium, implying a distinct displacement of nerve fibers. The vasa nervorum pattern was also altered in compressed sciatic nerves compared to the control group. Intense myelin sheath degeneration was also observed at the entrapment site and mainly in the distal segment with an influx of fibroblasts and Schwann cells to the lesioned regions (Figure 1). We also observed loosely textured and whorled cell-sparse structures, ranging from 15 to $145 \mu \mathrm{m}$ in diameter, found in the subperineurial space of the compressed sciatic nerve. In these areas we often observed isolated nerve fibers surrounded by collagen fibers. Some of these structures have a morphology suggestive of Renaut bodies (nerve cell-sparse structures with a predilection for sites of nerve compression and mechanical stress) $(7,8)$.

The number of myelinated axons in each nerve segment - control, proximal, entrapment or distal - was analyzed according to axonal position either in the central or marginal region of the nerve. Data are reported as percentage of the respective control region (Figure 2). Interestingly, the results observed at the entrapment site displayed a remarkable increase in the number of small axons either in the central $(26.36 \pm 12.48 \%)$ or marginal (53.54 $\pm 8.92 \%)$ nerve region in comparison with the axonal number in the control group. In addition, the distal segment of the compressed nerve presented a distinct decrease in axon number in both the central $(34.29 \pm 4.44 \%)$ and marginal $(24.04 \pm$ $3.68 \%)$ entrapped nerve regions comparatively to control nerves.
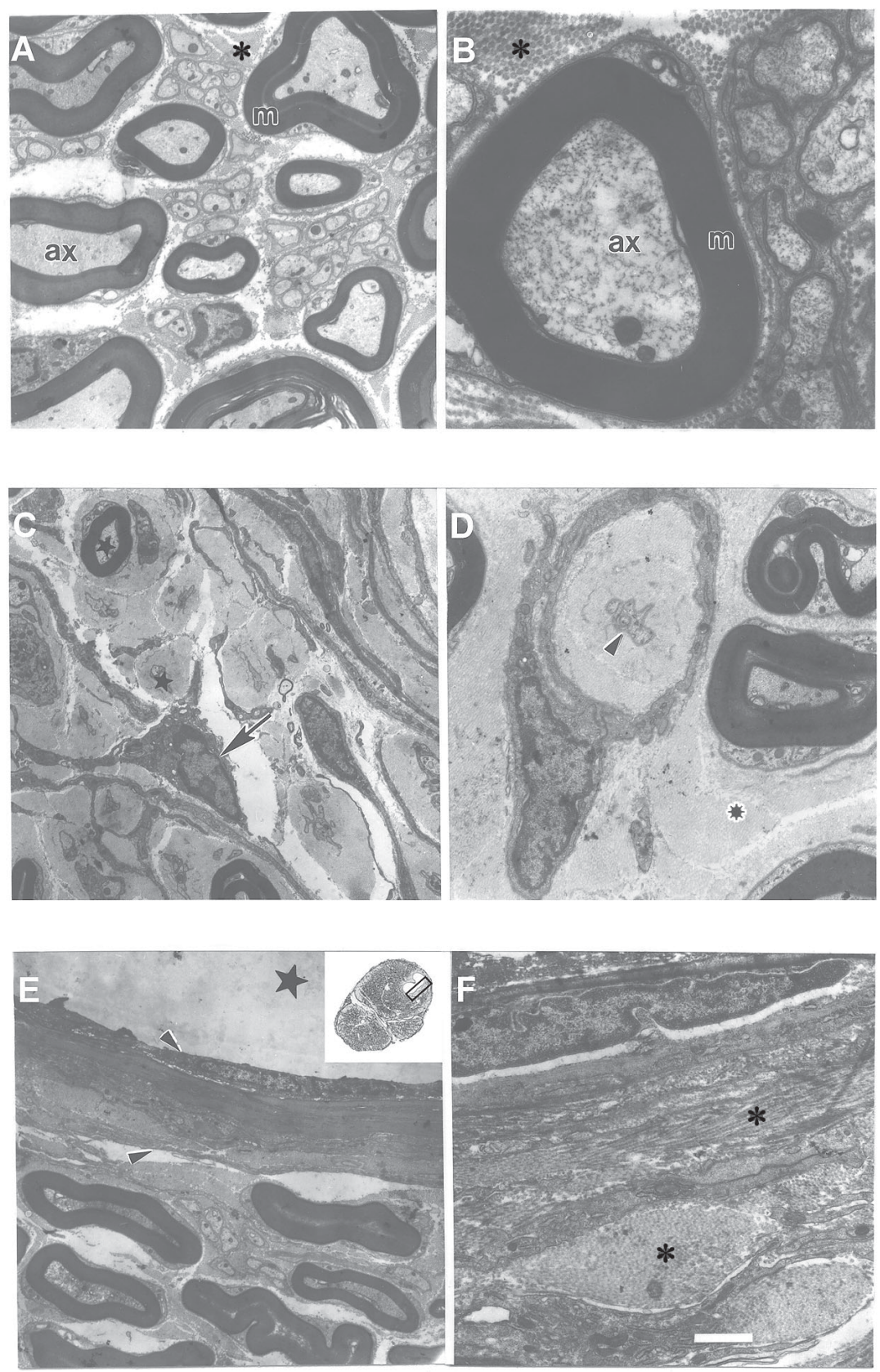

$A$ and $B$, Control: normal myelinated and unmyelinated nerve fibers. Observe collagen fibrils in the endoneurium (asterisks) and normal looking axoplasm (ax) and myelin sheath (m). $C$ and $D$, Compression area. $C$, Isolated nerve fibers (stars) are seen inside islands of collagen fibrils surrounded by fibroblasts (arrow). $D$, Higher magnification of one of these islands exhibiting a central nerve fiber devoid of myelin (arrowhead). Note thickening of the endoneurium (asterisk). E, Renaut body showing the lumen filled with amorphous material (star) and thickened wall (region between arrowheads). Observe compression of myelinated nerve fibers. The inset depicts a low magnification of a light microscopic view of a cross-sectioned nerve presenting a Renaut body. A rectangle signals the border of this Renaut body from which the electron micrograph shown in $E$ was obtained. $F$, Wall of a Renaut body lined with an elongated cell and exhibiting layers of collagen fibers arranged in various directions (asterisks). Value of magnification bar for each panel: $A=2.4 \mu \mathrm{m} ; \mathrm{B}=0.6$ $\mu \mathrm{m} ; \mathrm{C}=3.6 \mu \mathrm{m} ; \mathrm{D}=1.6 \mu \mathrm{m} ; \mathrm{E}=3.6 \mu \mathrm{m}$, and $\mathrm{F}=0.9 \mu \mathrm{m}$. 
Figure 2. The number of myelinated axons in each nerve segment - control, proximal, entrapment or distal - was analyzed according to axonal position either in central or marginal regions of the nerve after 5 weeks of survival. Data are reported as percentage of the respective control region. The number of myelinated axons in the respective regions of the control nerve was assumed to be $100 \%$. Data are reported as means \pm SEM All symbols placed on top of the bars represent statistically different groups $(P<0.01)$ compared with respective control region (ANOVA followed by post hoc Duncan multiple range test).

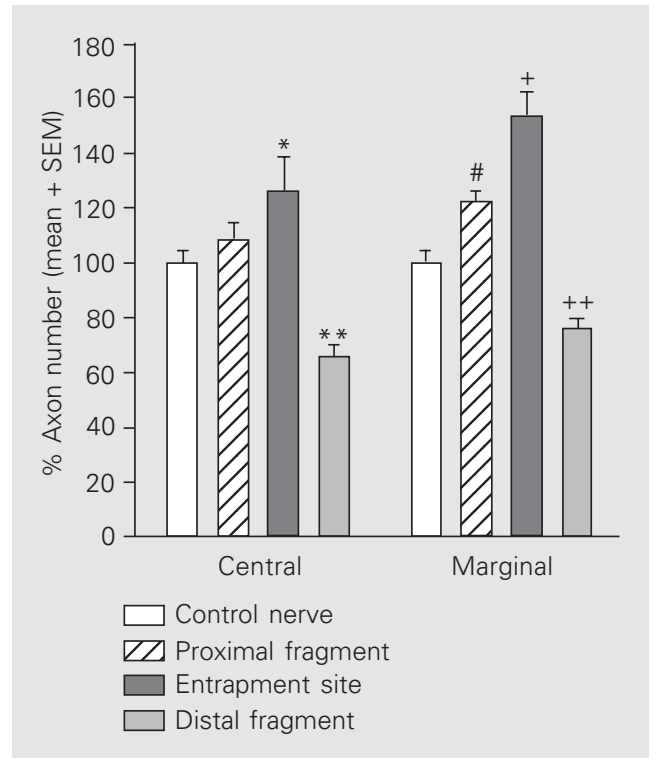

In the entrapment segment, we observed neural structures with a morphology highly suggestive of Renaut bodies. These bodies contained a loosely textured amorphous and fibrillar material (9-11). Renaut bodies exhibit mostly fibroblasts of perineurial and/or endoneurial origin with the extracellular matrix comprised of collagen fibrils, basal lamina material, and oxytalan filaments (10). Renaut bodies seem to be derived mainly from primary connective tissue elements of the endoneurium or from the degeneration of formed endoneurial structures such as capillaries. The latter possibility emerged from the study of human nerves showing a close association between thickened endoneurial capillaries and Renaut bodies (12). We observed similar changes in the hamster sciatic nerve. Histologic characteristics that differentiate Renaut bodies from malignant neurotropic infiltration are: a) a cell-sparse mass, b) absence of nuclear atypia, c) a less extensive than expected inflammatory infiltrate, and d) well-defined borders (10).

This model of experimental chronic entrapment caused a remarkable increase of axons of small diameter (less than $3 \mu \mathrm{m}$ ) at the site of compression. This finding may be notably attributable to axonal sprouting in response to nerve compression. This biological phenomenon was more evident in the marginal region, where it could be statistically confirmed both in the proximal and in the entrapment nerve segment. In addition, we observed an important axonal degeneration occurring at the distal segment both in central and in marginal regions that may be considered as a probable direct consequence of mechanical stress upon the sciatic nerve affecting its normal physiology. The present study has proven the usefulness of this experimental model in the production of clearcut histopathologic findings observed in entrapped sciatic nerves of adult hamsters. The entrapment procedure used in this investigation produces the formation of recognizable neuropathological signs $(13,14)$ at a faster rate than previously described for the compressed sciatic nerve of the rat (15). In addition, entrapped nerves exhibited Renaut bodies at the sites of nerve compression. Further investigations are necessary in order to clarify some of the neurobiological phenomena involved in the appearance of these abnormal structures in compressed nerves.

\section{Acknowledgments}

We thank Adiel Batista Nascimento, Antonia Lima Carvalho, Elizabeth Cunha Pena de Moraes and Jorge Luís da Silva for excellent technical assistance.

\section{References}

1. Harrison MJG (1976). Pressure palsy of the ulnar nerve with prolonged conduction block. Journal of Neurology, Neurosurgery and Psychiatry, 39: 96-99.

2. Rydevik B, McLean WG \& Sjostrand J (1980). Blockage of axonal transport induced by acute graded compression of the rabbit vagus nerve. Journal of Neurology, Neurosurgery and Psychiatry, 43: 690698.

3. Dahlin LB, Rydevik B \& McLean WG (1984). Changes in fast axonal 
transport during experimental nerve compression at low pressures. Experimental Neurology, 84: 29-36.

4. Jefferson D \& Eames RA (1979). Subclinical entrapment of the lateral femural cutaneous nerve. Muscle and Nerve, 2: 145-154.

5. Thomas PK (1963). The connective tissue of peripheral nerve; an electron microscope study. Journal of Anatomy, 97: 35-42.

6. Neary D, Ochoa J \& Gilliatt RW (1975). Sub-clinical entrapment neuropathy in man. Journal of the Neurological Sciences, 24: 283298.

7. Renaut J (1881). Récherches sur quelque points particuliers de I'histologie des nerfs. Archives de Physiologie Normale et Pathologique, 8: 161-190.

8. Ortman JA, Sahenk Z \& Mendell JR (1983). The experimental production of Renaut bodies. Journal of the Neurological Sciences, 62: 233-241.

9. Weis J, Alexianu ME, Heide G \& Schroder JM (1993). Renaut bodies contain elastic fiber components. Journal of Neuropathology and Experimental Neurology, 52: 444-451.

10. Skidmore RA, Woosley JT \& Tomsick RS (1996). Renaut bodies.
Benign disease process mimicking neurotropic tumor infiltration. Dermatologic Surgery, 22: 969-971.

11. Elcock LE, Stuart BP, Hoss HE, Crabb K, Millard DM, Bopp B, Mueller RE, Hastings TF \& Lake SG (2001). Renaut bodies in the sciatic nerve of beagle dogs. Experimental and Toxicologic Pathology, 53: 19-24

12. Asbury AK (1973). Renaut bodies. A forgotten endoneurial structure. Journal of Neuropathology and Experimental Neurology, 32: 334-343.

13. MacKinnon SE, Dellon AL, Hudson AR \& Hunter DA (1986). Chronic human nerve compression - a histological assessment. Neuropathology and Applied Neurobiology, 12: 547-565.

14. Campbell WW (1997). Diagnosis and management of common compression and entrapment neuropathies. Neurologic Clinics, 15: 549-567.

15. O'Brien J, MacKinnon S, MacLean A, Hudson A, Dellon A \& Hunter $D$ (1987). A model of chronic nerve compression in the rat. Annals of Plastic Surgery, 19: 430-435. 